\section{Acknowledgments}

Work in the authors' laboratory is funded by the Ministerio de Economía y Competitividad, the Innovative Medicines Initiative, the European Commission Seventh Framework Programme, and the Beta Cell Biology Consortium (National Institute of Diabetes and Digestive and Kidney Diseases).

Address correspondence to: Jorge Ferrer, Centre Esther Koplowitz, Villarroel 153, Barcelona, 08036, Spain. Phone: 34.93.227.5400 ext. 4542; Fax: 34.93.451.6638; E-mail: jferrer@clinic.ub.es.

1. Zaret KS, Grompe M. Generation and regeneration of cells of the liver and pancreas. Science. 2008;322(5907):1490-1494

2. Servitja JM, Ferrer J. Transcriptional networks controlling pancreatic development and beta cell function. Diabetologia. 2004;47(4):597-613.

3. Oliver-Krasinski JM, Stoffers DA. On the origin of the beta cell. Genes Dev. 2008;22(15):1998-2021.

4. Kroon E, et al. Pancreatic endoderm derived from human embryonic stem cells generates glucoseresponsive insulin-secreting cells in vivo. Nat Biotechnol. 2008;26(4):443-452.
5. Zhou Q, Brown J, Kanarek A, Rajagopal J, Melton DA. In vivo reprogramming of adult pancreatic exocrine cells to beta-cells. Nature. 2008; 455(7213):627-U630.

6. Jorgensen MC, Ahnfelt-Ronne J, Hald J, Madsen OD, Serup P, Hecksher-Sorensen J. An illustrated review of early pancreas development in the mouse. Endocr Rev. 2007;28(6):685-705.

7. Piper $\mathrm{K}$, et al. Beta cell differentiation during early human pancreas development. J Endocrinol. 2004;181(1):11-23.

8. Jonsson J, Carlsson L, Edlund T, Edlund H. Insulinpromoter-factor 1 is required for pancreas development in mice. Nature. 1994;371(6498):606-609.

9. Offield MF, et al. PDX-1 is required for pancreatic outgrowth and differentiation of the rostral duodenum. Development. 1996;122(3):983-995.

10. Gao N, LeLay J, Vatamaniuk MZ, Rieck S, Friedman JR, Kaestner KH. Dynamic regulation of Pdx1 enhancers by Foxa1 and Foxa2 is essential for pancreas development. Genes Dev. 2008;22(24):3435-3448.

11. Nelson SB, Schaffer AE, Sander M. The transcription factors $\mathrm{Nkx} 6.1$ and $\mathrm{Nkx} 6.2$ possess equivalent activities in promoting beta-cell fate specification in $\mathrm{Pdx} 1+$ pancreatic progenitor cells. Development. 2007;134(13):2491-2500.

12. Jacquemin P, Yoshitomi H, Kashima Y, Rousseau GG, Lemaigre FP, Zaret KS. An endothelial-mesenchymal relay pathway regulates early phases of pancreas development. Dev Biol. 2006;290(1):189-199.

13. Allen HL, et al. GATA6 haploinsufficiency causes pancreatic agenesis in humans. Nat Genet. 2011;44(1):20-22.

14. D'Amato E, et al. Genetic investigation in an Italian child with an unusual association of atrial septal defect, attributable to a new familial GATA4 gene mutation, and neonatal diabetes due to pancreatic agenesis. Diabet Med. 2010;27(10):1195-1200.

15. Carrasco M, Delgado I, Soria B, Martín F, Rojas A. GATA4 and GATA6 control mouse pancreas organogenesis. J Clin Invest. 2012;122(10):3504-3515.

16. Xuan S, et al. Pancreas-specific deletion of mouse Gata 4 and Gata 6 causes pancreatic agenesis. J Clin Invest. 2012;122(10):3516-3528.

17. Kuo CT, et al. GATA4 transcription factor is required for ventral morphogenesis and heart tube formation. Genes Dev. 1997;11(8):1048-1060.

18. Morrisey EE, et al. GATA6 regulates HNF4 and is required for differentiation of visceral endoderm in the mouse embryo. Genes Dev. 1998; 12(22):3579-3590.

19. Martinelli P, Canamero M, del Pozo N, Madriles F, Zapata A, Real FX. Gata6 is required for complete acinar differentiation and maintenance of the exocrine pancreas in adult mice. Gut. In press.

20. Molkentin JD. The zinc finger-containing transcription factors GATA-4, -5 , and -6 . Ubiquitously expressed regulators of tissue-specific gene expression. J Biol Chem. 2000;275(50):38949-38952.

21. Krapp A, et al. The bHLH protein PTF1-p48 is essential for the formation of the exocrine and the correct spatial organization of the endocrine pancreas. Genes Dev. 1998;12(23):3752-3763.

\title{
Defending the cornea with antibacterial fragments of keratin
}

\author{
Michael Zasloff
}

Georgetown Transplant Institute, Georgetown University School of Medicine, Washington, DC, USA.

\begin{abstract}
In addition to its role in refraction, the cornea forms a barrier between the eye and environmental and infectious insults. Corneal infections are surprisingly rare, suggesting that multiple aspects of the immune system are at play in mediating protection. In this issue of the JCI, Tam et al. describe the unexpected role of a structural protein, cytokeratin $6 \mathrm{~A}$, in this process.
\end{abstract}

The usual healthy appearance of the cornea and conjunctivae of the human eye should puzzle you. Why is it that this surface looks so healthy, most of the time? How is it that despite the almost certain diversity of microbes that come in contact with it, we so rarely see infection, or its associated sign, inflammation, evidence of the body's mechanisms that are called forth to fight off microorganisms? Lysozyme in tears can defend the eye, but organisms that inhabit the upper airway, such as Staphylococcus aureus are resistant to this enzyme

Conflict of interest: The author has declared that no conflict of interest exists.

Citation for this article: J Clin Invest. 2012; 122(10):3471-3473. doi:10.1172/JCI65380.
(1), and other antimicrobial systems must be at work, because in the various dry-eye syndromes, the reduced tear production is not associated with frequent bacterial infections. More perplexing is the fact that a corneal transplant will not necessarily develop infections at the incision or around the suture tracks, and antibiotics are not necessarily required postoperatively (2). Thus, even the wounded cornea seems to handle microbes in some mysterious and remarkably effective - fashion.

\section{Layers of protection}

The cornea is a wonderful, close-up example of a site protected almost completely by the chemical and physical defenses of our innate immune system. Tears contain high concentrations (about 1 to $2 \mathrm{mg} / \mathrm{ml}$ ) of each of three antimicrobial proteins: lysozyme, lactoferrin, and lipocalin (3). The presence of lactoferrin, which chelates iron, and lipocalin, which captures the iron-transporting siderophores used by many bacteria, tells us that many microbes would discover the microenvironment of the corneal epithelium to be unfavorably iron-depleted. The corneal epithelium secretes several types of mucin that adhere to the corneal surface (4) and form a barrier that both provides a physical shield from invaders and creates an "unstirred" micron-thick fluid layer between itself and the corneal epithelial cell. Antimicrobial peptides are secreted into this barrier and can accumulate without diffusion (or dilution) into to the tear fluids. Furthermore, we have known for some time that the epithelium expresses several wellcharacterized antimicrobial peptides, both constitutively and induced following injury (5). These include several of the $\beta$-defensins and LL-37 (cathelicidin), which are believed 


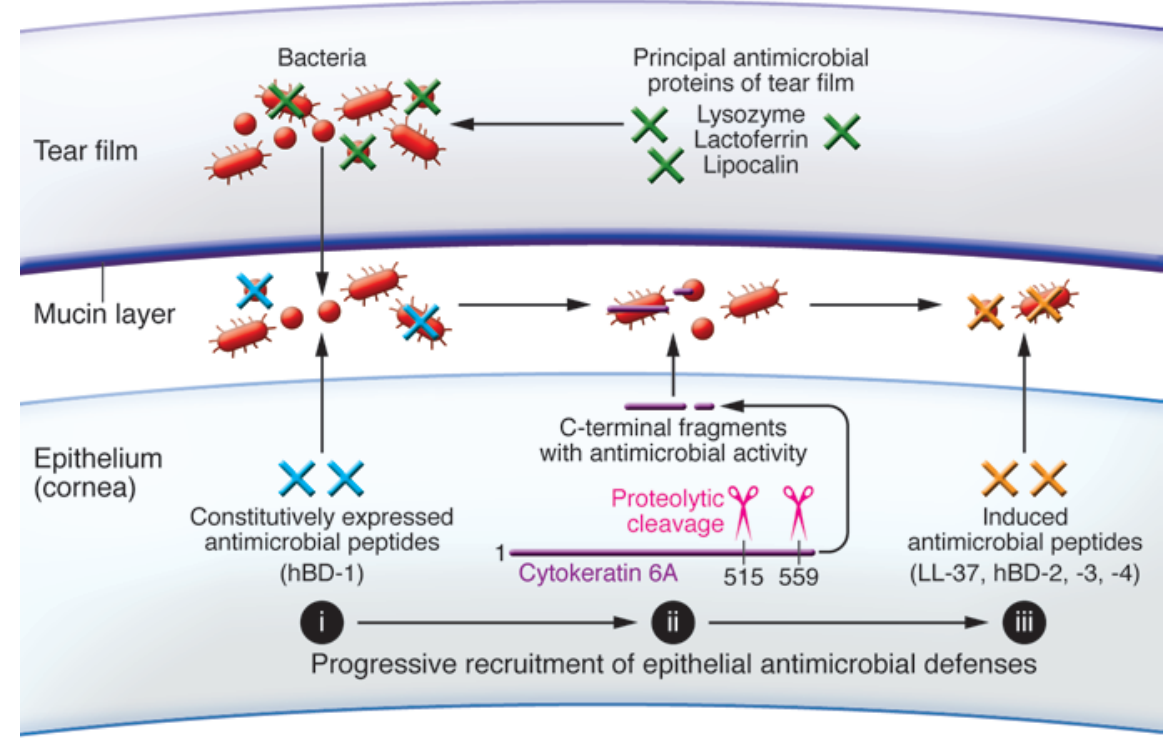

\section{Figure 1}

Principal antimicrobial defenses of the human eye. The epithelium of the cornea is depicted with an overlying mucus layer. A hypothetical progressive sequence is presented in which successive antimicrobial defenses are recruited. The tear film proteins, lysozyme, lactoferrin, and lipocalin create an environment that is nonoptimal for microbial growth. The mucin layer, derived from mucins secreted by the epithelium, creates a physical barrier that microbes must overcome in order to invade the cornea. (i) Should a microbe penetrate the mucin layer, it faces constitutively expressed antimicrobial peptides, such as human $\beta$-defensin-1 (hBD-1). (ii) If the microbe gains access to the epithelium, we imagine that cytokeratin fragmentation, as described by Tam et al., generated by cleavage of a preexisting abundant protein, is rapidly mobilized. (iii) Expression of a battery of antimicrobial peptides is subsequently induced over the course of hours.

to be secreted from the epithelial cell onto the corneal surface beneath the mucin layer (6). Antimicrobial peptides act by binding to microbial membranes and fatally disturbing their permeability (7). An organism that somehow made it through the mucin layer would then encounter the high concentrations of antimicrobial peptides bathing the surface of corneal epithelial cells, be killed rapidly, and fail to adhere or to penetrate.

\section{Finding a new player}

In this issue of JCI, Tam et al. describe another antimicrobial defense of the corneal epithelium that has gone unnoticed to date (8). These investigators hypothesized that there might be additional, as yet undiscovered, antimicrobial peptides being expressed by the human corneal epithelium. To search for these putative peptides, they derived an immortal cell line from human corneal epithelium and differentiated it into mature corneocytes by addition of calcium ions. In vitro, a crude extract of these cultured cells exhibited antibacterial activity against Pseudomonas aeruginosa. Fractionation of the extract on the basis of molecular weight demonstrated that the activity resided in two fractions (less than 3,000 Da and between 3000 and 10,000 $\mathrm{Da})$. Analysis of these fractions by LC/MS identified the principal active molecules to be peptide fragments derived from the carboxyl-terminal region of cytokeratin 6A.

To confirm that cytokeratin $6 \mathrm{~A}$ was the protein from which these peptides originated, Tam et al. demonstrated that reducing cytokeratin $6 \mathrm{~A}$ expression using siRNA significantly attenuated antibacterial activity (8).

The keratin peptides identified in the extract were synthesized, and their individual activities were studied. Perhaps the most surprising result (for those interested in the details of antimicrobial peptide structure-function) was that several of these short peptides did not exhibit the anticipated sequence and structural characteristics of classical linear antimicrobial peptides, because they were glycine-rich and lacking both a net cationic charge and the tendency to form an $\alpha$-helix. Several of these peptides exhibited bactericidal activity in vitro against potential ocular pathogens in addition to $P$. aeruginosa, including S. aureus and Streptococcus pyogenes.
One peptide, a 19-mer with a "classical" cationic amphipathic sequence, was effective in rapidly killing a cytotoxic strain of $P$. aeruginosa in either water or at physiological ionic conditions (150 $\mathrm{mM} \mathrm{NaCl})$, suggesting it would retain activity within the liquid film in contact with the apical surface of the corneal epithelium. This peptide, when introduced into culture medium, effectively protected corneal epithelial cells from invasion and cytotoxicity by $P$. aeruginosa. Further studies by Tam et al. suggested that this 19-mer bound specifically to the bacterial cytoplasmic membrane and caused it to become leaky, subsequently killing the bacterial cell within minutes of exposure.

Perhaps most remarkably, Tam et al. demonstrated that subcorneal injection of cytokeratin 6A siRNA into the cornea of a live mouse, reducing expression of cytokeratin $6 \mathrm{~A}$ by about $25 \%$, resulted in a 5 -fold increase in the adherence of $P$. aeruginosa introduced onto the treated cornea.

The conclusion from these studies is that a cytokeratin, a protein that plays a structural role within cells, appears to protect the surface of the eye. By some mechanism, not as yet understood, fragments of this protein are generated that find their way to the surface of the cornea and kill microbes.

\section{A new role for structural proteins?}

Precedents for structural intracellular proteins serving extracellular antimicrobial functions in vertebrates exist. A notable example has been described in the stomach of the Asian toad (9). The 39-amino acid antimicrobial peptide buforin is the $\mathrm{N}$-terminal fragment of histone 2A that appears to play an antimicrobial role on the gastric mucosa of this species. Buforin is produced by the action of gastric pepsin, which acts on unacetylated cytoplasmic histone 2A that has been secreted into the gastric lumen (9).

The antimicrobial defense system described by Tam et al. has the capacity to be rapidly mobilized, since it uses an abundant protein already present within the cell. In contrast, the expression of the induced antimicrobial peptides would likely occur over the course of hours. Thus, this system might be critical in the initial phases of microbial assault (Figure 1). Many questions are provoked by this discovery. What mechanisms are involved in the fragmentation of cytokeratin 6A, and what are the proteases involved in the process? Where within the corneal epi- 
thelial cell are the fragments generated? What signals the fragmentation and/ or secretion of these fragments? Since the various keratin fragments appear to have different antimicrobial specificities, might assault by different bacteria result in different patterns of fragmentation? Furthermore, cytokeratin $6 \mathrm{~A}$ is present in many other sites in the body exposed to microbes, such as the skin, hair, teeth, and various mucosal surfaces, and it is unclear how widely used this keratinbased antimicrobial defense might be. Hopefully, some of these questions, and others not posed, will be answered in the future. In addition, as Tam et al. suggest (8), these keratin-derived antimicrobial peptides appear to be exciting new biocompatible candidates for development as human anti-infective therapeutics.

Address correspondence to: Michael Zasloff, Professor of Surgery and Pediatrics, Transplant Institute, Georgetown University Hospital, 2 Main, 3800 Reservoir Road NW, Washington, DC 20007, USA. Phone: 484.433.7807; Fax: 202.687.2792; E-mail: maz5@georgetown.edu.

\footnotetext{
1. Bera A, et al. Influence of wall teichoic acid on lysozyme resistance in Staphylococcus aureus. J Bacteriol. 2007;189(1):280-283.

2. Liu Y, et al. Topical application of FTY720 and cyclosporin A prolong corneal graft survival in mice. Mol Vis. 2012;18:624-633.

3. Yamada M, Mochizuki H, Kawai M, Tsubota K,
}

Bryce TJ. Decreased tear lipocalin concentration in patients with meibomian gland dysfunction. $\mathrm{BrJ}$ Ophthalmol. 2005;89(7):803-805.

4. Mantelli F, Argueso P. Functions of ocular surface mucins in health and disease. Curr Opin Allergy Clin Immunol. 2008;8(5):477-483.

5. McDermott AM. The role of antimicrobial peptides at the ocular surface. Ophthalmic Res. 2009;41(2):60-75.

6. Jäger K, Nielitz A, Garreis F, Sel S, Nave H, Paulsen FP. In vitro evidence of involvement of the epithelial $y+$ transporter in beta-defensin production on the ocular surface. Ann Anat. 2011;193(6):479-485.

7. Zasloff M. Antimicrobial peptides of multicellular organisms. Nature. 2002;415(6870):389-395.

8. Tam C, Mun JJ, Evans DJ, Fleiszig SMJ. Cytokeratins mediate epithelial innate defense through their antimicrobial properties. J Clin Invest. 2012; 122(10):3665-3677.

9. Kim HS, et al. Pepsin-mediated processing of the cytoplasmic histone H2A to strong antimicrobial peptide buforin I. J Immunol. 2000;165(6):3268-3274.

\title{
Looking in the miR-ror: TGF- $\beta$-mediated activation of NF-kB in glioma
}

\author{
Christine E. Eyler ${ }^{1}$ and Jeremy N. Rich ${ }^{2,3}$ \\ 1Department of Internal Medicine, Brigham and Women's Hospital, Boston, Massachusetts, USA. \\ 2Department of Stem Cell Biology and Regenerative Medicine, Lerner Research Institute, Cleveland Clinic, Cleveland, Ohio, USA. \\ ${ }^{3}$ Department of Molecular Medicine, Case Western Reserve University School of Medicine and University Hospitals, Cleveland, Ohio, USA.
}

\begin{abstract}
The explosive growth in our understanding of the molecular underpinnings of glioblastomas has served as an instructive paradigm for other cancers. However, the exact nature by which many of the pathogenic drivers connect is less well known, and elucidation of relationships between critical genetic and signaling alterations may inform the development of therapeutic approaches to the disease. In this issue, Song et al. identify miR-182 induction as a mechanism by which TGF- $\beta$ stimulation aberrantly activates NF- $K B$ signaling in glioblastoma cells, clarifying a critical point of crosstalk between molecular signaling pathways. Their findings provide a greater understanding of the complex interplay between signaling pathways in cancer that may ultimately prove useful in the development of synergistic targeting approaches.
\end{abstract}

Glioblastoma (World Health Organization grade IV glioma) is the most prevalent primary brain tumor; these highly lethal cancers are characterized by alterations in multiple critical intracellular signaling networks as well as by inactivation of tumor suppressors (1). Although specific pathways and molecules are frequently hyperactive and appear dominant in glioblastoma, unilateral molecular targeting approaches have been disappointing clinically. For example, most glioblastomas dis-

Conflict of interest: The authors have declared that no conflict of interest exists.

Citation for this article: $J$ Clin Invest. 2012; 122(10):3473-3475. doi:10.1172/JCI66058. play hyperactive EGFR signaling as a result of increased receptor copy number or oncogenic activating mutations. However, single-agent EGFR targeting has not been successful in clinical trial (2). Because glioblastoma cells display plasticity in signaling networks without addiction to any one oncogene, successful therapy will require multipronged approaches that impede various active pathways for success with molecularly targeted agents (3). In theory, identification of signaling keystones and their interactions within the structurally complex architecture of glioblastoma will inform the development of effective therapeutic approaches to topple the colossus of cancer signaling.

\section{Mapping the signaling axes}

The multiple concerted signaling alterations that contribute to the malignant characteristics of glioblastoma have been interrogated by many researchers. NF- $\mathrm{\kappa B}$ pathway activation has emerged as one of the critical central signaling axes in glioblastoma cells. NF- $\mathrm{\kappa B}$ signaling can be activated by EGFR signaling, which is often a key feature of gliomas (4). Similarly, the constitutively active EGFRvIII mutant often present in glioblastoma acti-

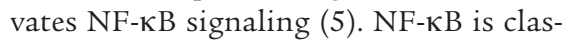
sically activated by inflammatory-related mechanisms, which also may be present and of oncogenic importance (6). In addition, recent work demonstrates that deletion of NF-KB inhibitor- $\alpha$ (NFKBIA, which encodes IкB $\alpha$ ) in glioblastomas with nonamplified EGFR is associated with shorter patient survival, which suggests that NF-KB signaling is a central node in oncogenic signaling both in EGFR-amplified and nonamplified glioma (7). It is worth mentioning that signaling via other activated receptor tyrosine kinases (RTKs) can also stimulate NF- $\mathrm{KB}$ signaling. Thus, NF- $\mathrm{KB}$ is a central pathway mediating the effects of mitogen-activated signaling pathways like PDGFRA, ERBB2, and MET (1). 